\title{
Beaming into the News: \\ A System for and Applications of Tele-Immersive Journalism
}

\author{
Sameer Kishore ${ }^{1}$, Xavi Navarro$^{1}$, Eva Dominguez ${ }^{2,4}$, Nonny De La Peña ${ }^{3,4}$, Mel Slater ${ }^{1,5,6 *}$ \\ 1 Event Lab, Faculty of Psychology, University of Barcelona, Spain \\ 2 Department of Communication, Universitat de Pompeu Fabra, Barcelona, Spain \\ 3 Annenberg School of Journalism and Communications, University of Southern California, \\ USA. \\ 4 Independent Journalist \\ 5 Department of Computer Science, University College London, UK. \\ 6 Institució Catalana de Recerca i Estudis Avançats (ICREA), Barcelona, Spain. \\ *Corresponding author: \\ Mel Slater \\ Event Lab, \\ Universitat de Barcelona, Facultat de Psicologia, \\ Campus de Mundet - Edifici Teatre, \\ Passeig de la Vall d'Hebron 171, \\ 08035 Barcelona \\ Spain \\ Tel: +34934039618 \\ Email: melslater@ub.edu
}

\section{Keywords}

I.3.7.g Virtual reality; I.2.9 Robotics; I.2.9.c Commercial robots and applications; L.2.0.r Telemanipulation; L.2.0.q Telepresence

\begin{abstract}
We show how a combination of immersive virtual reality and robotics can be used to beam a physical representation of a person to a distant location, and an application of this system in the context of journalism. Full body motion capture data of a person is streamed and mapped in real time, onto the limbs of a humanoid robot present at the remote location. A pair of cameras in the robot's 'eyes' stream stereoscopic video back to the HMD worn by the visitor, and a two-way audio connection allows the visitor to talk to people in the remote destination. By fusing the multisensory data of the visitor with the robot, the visitor's 'consciousness' is transformed to the robot's body. This system was used by a journalist to interview a neuroscientist and a chef 900 miles distant about food for the brain that was then published in the popular press.
\end{abstract}




\section{Introduction}

Ideas originally expressed in science fiction have often later been realized or have at least been an inspiration for research and technological advance. Perhaps the most famous example is the Star Trek 'communicator' as the conceptual forerunner of the cell phone. As another example, the finalists for a $\$ 10 \mathrm{M}$ competition to realize the Star Trek medical 'Tricorder' have recently been announced (URL 1). Here we consider another Star Trek concept - the idea of beaming instantaneously transporting someone from one physical location to another. While it may be impossible to teleport humans or solid matter instantaneously from one place to another a physical simulation can nevertheless be realized by exploiting current ideas in cognitive neuroscience combined with immersive virtual reality, teleoperator systems and robotics. In this paper we describe a system that makes this possible, and some examples of its use in the context of an application to journalism. Conceptually the work relies on two key ideas - telepresence (or presence), the illusion of being located in a place other than where the physical body is located [4], and illusory virtual body ownership [7].

The term most closely associated with the illusion of being present at a place that is different from a person's true location is 'telepresence' [8]. Additionally, along with telepresence, another key aspect for simulating teleportation requires a representation of the 'visitor' who is to appear at the remote location. A physical representation, such as a humanoid robot in the remote location that allows the visitor to interact with people and perform tasks affords a physical or embodied experience compared, for example, to tele-conferencing. Technologies that allow someone to remotely control a machine or a robot are referred to as teleoperation. The combination of teleoperation and telepresence has given rise to development of several 'telepresence robots', that allow people to interact with colleagues or attend meetings remotely [9]. However, as mentioned in [10], these robots can be thought of simply as video conferencing on wheels.

The premise of our work is that the visitor (see Inset 1) should have the perceptual illusion that their robot representation is their own body. This illusion of body ownership is influenced by several factors, including appearance of the virtual/remote body, agency, and a first-personperspective view from the eyes of the body [7]. Thus, to induce this feeling it is important to have a body that is humanoid, and to have a natural way of controlling this body: for example, through being able to motion capture the movements of the visitor and map this on to the limbs of the remote body in real time [11]. By combining all the aspects discussed above, consequently allowing someone to see a remote location from the perspective of a remote body (a humanoid robot), and have the ability to hear, touch, interact with people, and move the remote body naturally in the remote space as if it were their own, we can simulate a type of physical 
teleportation. In [6] we described our approach of developing systems and scenarios where this technology could be used in a meaningful way. In this paper we show how this technology can be utilized in the context of journalism - where either the journalist beams to a remote location embodied in a humanoid robot, or an interviewee beams to the location of the journalist.

Journalists have always needed to travel to remote or unusual locations in order to gain insight about a story. Having a "view from the ground," as described by veteran World War II reporter [12] gives a journalist the crucial authenticity to connect audiences to reports of unfolding scenarios, events or stories. However, at times locations are off-limits because of inherent physical dangers, such as when the violence of a war makes it too risky to deploy there or the conditions are too inhospitable for the human body, as during a major catastrophe such as a volcanic explosion or when exploring the ocean floor or space. The ability to travel to these places embodied in a robot that corresponds to the human shape and which can be driven in parallel with natural biological body movements can extend reporting in any type of condition or environment. Moreover, good journalism also requires an ability to connect with the individuals who are being interviewed. By creating robots with an appearance compatible to the circumstance, including gender, racial and age-appropriateness, the journalist will be able to side-step a common barrier of prejudice based on the instantaneous judgments made about physical and virtual appearance [13].

\section{Background}

\subsection{Telerobotics, Embodiment and Body-Ownership Illusion}

One of the first of the recent batch of new and relatively low cost telepresence robots is described in [14] called PRoP (Personal Roving Presence). It is a setup with a single video camera and a microphone, mounted on top of a pole that also has an LCD screen, which is attached to a drive base. Since then, many similar robots have been developed and manufactured commercially. Some robots have added features, such as the ability to express non-verbal cues, and have been shown to lead to a better quality of interaction [15]. A case study [10] compared the various telepresence robots currently on the market by placing them in social scenarios and observing how they were perceived by the people interacting with them. A commercially available telepresence robot allows the user to work remotely, controlling the robot and interacting with colleagues at the workplace by using a desktop-based application [9]. Another telepresence robot, built specifically for the medical field allows elderly people to communicate with healthcare professionals [16].

It is evident that since the origin of this concept, all subsequent advances have been similar to their predecessors in terms of basic shape, control and communication paradigms. The one aspect that is consistent among all of these types of telepresence robots developed to date is the 
way the robot is used by the person controlling it. In almost all the cases, the person has a desktop screen with either a joystick or a keyboard to control the robot. Although users have reported to feel presence, the key component missing in these types of system is the feeling of the body of the robot to be their own.

To illustrate this idea of body ownership consider the work of Petkova and Ehrsson [17] who described an experiment where a mannequin body was used to apparently substitute the real body of the participants. This was achieved by mounting a pair of cameras on top of the mannequin body looking down towards it. These cameras fed a stereo head-mounted display worn by the participants, so that when they looked down towards their real body they would see the mannequin body in its place. This led to participants experiencing the perceptual illusion that the mannequin body was their own, provided that tactile stimulation that was seen to be applied to the mannequin body was synchronously applied to their real body. Other studies have shown that although the appearance of the body matters, it is not crucial for invoking the illusion [18]. Results have shown that males can successfully have this illusion in virtual reality with respect to a female body [7], thin men with respect to a fat body [19], adults to a child body [20], lightskinned people to a dark-skinned body [21-23], and even with respect to a highly asymmetrical body [24]. However, it appears that the body has to have human characteristics for this illusion to occur [25]. This is a promising result, since even though the humanoid robot that we have used for beaming has a morphology resembling that of a human it is nevertheless robotic in appearance. Furthermore, it has been shown in another study that this humanoid robot can be used to successfully induce the illusion of body ownership [26]. The effect of varying visual perspective, visuomotor correlation and agency have all been studied in detail [27,28], and it has been shown that a visuomotor correlation has a strong influence on inducing the illusion of ownership [20, 29-31] and this has also been used successfully in robotic embodiment [32]. However, in [33] it was found that changing perspective affects the body ownership in an android robot, and was shown that it can be induced using either first-person or third-personperspective.

Affording visitors to see through the 'eyes' of the robot, and providing them with a natural and congruent method of controlling the limbs of a humanoid robot, we increase the likelihood of a high sense of agency and body ownership with respect to the remote robot body, based on research into body ownership and agency in virtual reality [30, 31]. Hence in our system the visitor may not only feel present in the remote location, but can also have the illusion of ownership over the physical body in the remote destination, which they can use to interact with people, move around and manipulate the remote environment as if they were attending with their biological body. Thus, not only does the robot provide a physical representation of the person, it also acts as a surrogate body that is driven by the visitors as if it were their real body. 


\section{Inset 1: Beaming}

Beaming involves transmitting a digital representation of a person from one place to another, but where they can be physically embodied in the remote place. Applications from various fields have been developed that have exploited this technology, such as acting rehearsals [1, 2], teaching music remotely and medical rehabilitation [3]. It has even supported the teleportation of a human into the cage of a rat, allowing real-time interaction between rat and human, each at their own scale [5]. The following terms, defined in the context of the project, have been used throughout the rest of this paper [6]:

- Visitor: The person who 'travels' to the remote location.

- Transporter: This is the system used to 'beam' the visitor - requiring a highresolution wide field-of-view and head-tracked head mounted display (HMD), fullbody tracking systems and high-end audio devices. This technology is required to capture multisensory data of the visitor, and transmit it to the destination, as well as to digitally display the destination to the visitor.

- Destination: The destination is the remote physical location to where the visitor is transported. Here, it is required for the visitor to be represented in some way, for example, as a humanoid robot.

- Locals: The people present in the remote destination who can interact with the visitor are referred to as locals. Ideally the locals should not be encumbered by any equipment in order to be able to see and interact with the remote visitor.

\section{Materials and Methods}

\subsection{The Transporter}

The technology involved in the Transporter can be considered from two points of view. The first is the technology required to display the remote destination at the Transporter. To this effect, a stereoscopic 3D video feed from two cameras separated by a standard interocular distance at the destination is streamed via the Internet to the HMD in real-time. The HMD that has been used for the various applications of this system is a stereo NVIS nVisor SX111, although the Oculus Rift has also been used. The audio captured from the destination is also streamed to the transporter in real-time and played back through high quality headphones.

The second aspect concerns transferring the visitor's behavior to the humanoid robot representing the visitor at the destination. To provide the visitor with the most natural method of controlling the robot, the limb motions of the visitor are tracked in real-time. The head of the 
visitor is also tracked with 6 degrees of freedom by an Intersense 900, which is attached to the HMD itself, while the body can be tracked by a variety of full-body tracking systems. For our system, we have used the Xsens Inertial Motion Capture system most frequently, although other commercially available systems such as Arena Optitrack or Microsoft Kinect can also be used. These tracking systems can be used to capture the position and orientation of each limb of the visitor in real-time. Additionally, they also supply spatial information of the visitor with respect to the world. This enables us to not only track each limb, but also to observe the movements of the visitor in 3D space inside the transporter. Once we have this information, it is used as an input for a system that was developed specifically to convert this tracking data and map it to the humanoid robot at the destination, in real time [11]. This system is also what differentiates this setup from other telepresence arrangements, as it facilitates a much more natural method of controlling the robot. Instead of using a desktop screen with a joystick or keyboard, participants have the ability to move the robot's body as they would move their real body. The software application that manages all the data exchange and the rendering in the HMD has been programmed in the XVR system [34].

\subsection{The Destination}

At the destination, the visitor is represented by a robot through which he or she can interact with the locals in a natural way, and without encumbering the locals with the requirement to use or wear any special equipment. Hence, the essential component of the setup at the destination is the humanoid robot that acts as the substitute body for the visitor. The robot that we have used for the system is the Robothespian, manufactured by Engineered Arts, UK. This is a $180 \mathrm{~cm}$ tall humanoid robot, with two legs, a torso, two arms, and a head. The joints of the robot's upper limbs are pneumatic, while the torso and head, each with three degrees of freedom, move with a DC motor. To allow the visitor to see from the robot's perspective, two Microsoft HD-3000 webcams are mounted on the robot's forehead. The feed from these two cameras is streamed to the transporter, and rendered in the HMD of the visitor, who therefore can see the destination in stereo. Since the head of the robot is also directly mapped and controlled by the head movements of visitors they can also see the surrogate robot body when looking towards themselves from a first person perspective. Hence, if the visitor looks down, they see the robot body instead of their own, or looking at a mirror in the destination the visitor will see a reflection of the robot body which due to the real-time motion capture and mapping, follows their own movements as well. The robot also has a built-in speaker and an omnidirectional microphone that are used by a builtin Skype API for two-way audio communication between the transporter and destination. Furthermore, the Skype API also detects incoming audio, which is used for simple lip-syncing by moving the robot's lower jaw based on the amplitude of the incoming sound.

The lower half of the robot is fixed in place, thus, the robot cannot walk on its own. However, to facilitate translation and rotation of the robot in 2D space, a completely programmable platform was custom-built for this specific purpose, on which the robot was mounted. Using the system 
described in [11], new values for the position and orientation of each limb in the upper arm and head are sent to the robot at every frame. Additionally, the torso of the visitor is also tracked and this information is used to compute the movement of the visitor in 2D space, which is subsequently streamed to the platform concurrently. Figure 1 shows the schema for this system.

Using this system, the visitor is able to see the remote destination in stereoscopic $3 \mathrm{D}$, and has head-based sensorimotor contingencies since the head of the robot moves as the visitor moves their head. This contributes to provide a very rich multisensory experience and to the sense of presence in the remote location [35]. Furthermore, the movement of the arms of the visitor and the robot are congruent, which can also be seen by the visitor through the 'eyes' of the robot, engendering agency.

\section{Tele-Immersive Journalism Used for News about Tele-Immersive Journalism}

The system has been used several times for the purposes of tele-immersive journalism, and has been extensively demonstrated in the media. The BBC was the first news channel to cover the system, where they carried out an interview using the system itself (URL 2). The journalist was present at University College London (the destination). A Robothespian was located there that represented the visitor (interviewee), a neuroscientist who was physically located in Barcelona, Spain, and was 'beamed' to London, where she was interviewed about the system.

The system was also used by TV3 based in Catalonia and the Spanish newspaper La Vanguardia, where the relationship between interviewer and interviewee was reversed (URLs 3-4). This time, the two journalists were physically present in Barcelona, Spain, and were 'beamed' to University College London where they controlled the Robothespian, and conducted an interview about the system. The news article was printed in La Vanguardia while TV3 aired it as part of a science documentary.

\section{Tele-Immersive Journalism to Report News}

While the demonstrations mentioned above were a first of their kind and received widespread news and media coverage, the interviews had always been about the system itself. The first time that this setup was used by a journalist to not just experience or showcase the system, but to actually apply this technology for conducting interviews about other issues was by journalist Nonny de la Peña.

In a session that lasted for about three hours, Nonny de la Peña 'beamed' to Barcelona, Spain from Los Angeles, USA, and conducted two sets of interviews. The first was with an AIDS researcher whose team had recently discovered an important result in HIV research. The second was to conduct a debate amongst three students who were pro-, anti- or neutral about Catalonia's 
bid for independence from Spain. The debate was led and moderated by her remotely, i.e., as embodied in the robot. This event was broadcast live on Barcelona TV and an impromptu interview was also conducted where a journalist from BTV asked Nonny de la Peña, while embodied in the robot, about future applications of this technology (URL 5).

Nonny de la Peña utilized the system once again, when she beamed from London to Barcelona to conduct an interview with Dr. Perla Kaliman about her research and book regarding types of food and cooking that are good for the brain [36]. In this case the interview using this system was published in traditional news media. The article, which focused solely on the substantive issue of food for the brain, rather than the system that was used, was published in the newspaper Latino LA (URL 6).

In this case the visitor (the journalist) was physically present in University College London where we had set up a laboratory as a transporter system. The transporter was equipped with a high resolution, large field of view stereo NVIS nVisor SX111 HMD. The body of the visitor was tracked in real-time by the Xsens MVN motion capture suit, and an Intersense 900 head tracker was used to track the orientation of the visitor's head. The position and orientation values were processed using the algorithm described in [11] that in order to remap them to angles compatible with the robot's limbs in real time. High-quality headphones and microphone were used to facilitate two-way audio communication.

The destination was the University of Barcelona, Spain. A Robothespian with specifications as described in the materials section was used as the physical representation of the visitor. The speaker and microphone built in to the robot provided the necessary hardware for the audio communication. Additionally, a mirror was also placed in front of the robot. The setup explained above can be seen in Figure 2 and also shown in video http://youtu.be/ry6dmWB34qI.

The journalist Nonny de la Peña approached the task at hand with the same intention as when doing an interview with her biological body rather than conducting the conversation on the phone or through Skype. The interview questions were prepared prior to the event and included reading background information and exchanging emails as would typically be done in preparation for reporting any piece. On the day of the interview, prior to donning the Xsens motion capture suit, de la Peña reviewed her research and committed interview questions to memory.

Finally, de la Peña notes that her ability to report was enhanced in comparison to conducting interviews with current video-based technologies. Using the robot as an extension of self, de la Peña was able to shake hands, make eye contact or adjust viewpoint to address unique individuals as well as to 'walk over' and see materials provided by an interview subject. By using her biological body in a similar way as if she were actually present on scene, de la Peña 
had a freedom to engage with the interview subject in ways not possible with Skype or the telephone.

\section{Discussion}

We have developed and presented a novel system that significantly builds upon the existing standard telepresence robot setups by combining theoretical and practical knowledge from the fields of cognitive science and computer science. This system not only allows a person to immediately interact with people in a remote destination, but it also invokes a feeling of owning a physical body, thereby giving the illusion of really being there in a physical sense. Additionally, the method of controlling this body is direct - the robot limbs and torso are moved by moving the corresponding parts of the real body. Thus, the robot body can be used for gestures and integrating other forms of non-verbal communication in the experience as well. This substitution of the real body by a surrogate robotic body that allows someone to see, move and interact with the environment as they would if they were physically present at the destination.

The main bottleneck in terms of cost and accessibility are the hardware involved. Thanks to recent advances in the field of gaming hardware with the recent proliferation of low cost and high quality head-mounted displays at consumer prices some of the critical elements are likely to become widely available. Another consumer device that can be used to advantage is the Microsoft Kinect. The current version of the device is already capable of full-body real time tracking, and consequently, can be used in our system to drive the robot remotely. One advantage the Kinect has over Xsens is that the Kinect uses markerless tracking. The Xsens, which is based on inertial tracking, requires the visitor to put on a special suit and perform a pre-calibration every time. The Kinect on the other hand uses computer vision techniques to detect and track users without any additional equipment. By combining a good quality, reasonably priced and portable HMD with a markerless and portable full-body tracking system, we can set up a transporter practically anywhere, since the entire setup is highly compact. This is technically already possible by using an Oculus Rift with a Kinect, albeit with lower quality motion capture. However, there is still some way to go before we have low-cost life-sized humanoid robots available commercially and universally.

This system aims to provide a vision for the near future, where cities could have docking and charging stations of humanoid robots available for rent. People could instantly teleport to any part of the world by becoming embodied in the humanoid robot that they would select from the station, and interact with other people, or even other embodied robots without ever having to spend time, money and energy in travelling. This is the ultimate vision of beaming. 


\section{Acknowledgements}

This work was funded under the European FP7 Integrated Project BEAMING (contract number 248620). We thank Carlota Crusafon (UB) and Aitor Rovira (UCL) for helping to set up the transporter system at UCL and Evans Hauser for helping at the UB destination. We also thank Marie Schutgens and Bregtje van der Haak of VPRO Tegenlicht, and UBTV for providing film footage. We also thank Engineered Arts Ltd for the loan of a Robothespian at UCL, and NVIS for the loan of an NVIS SX111 at USC.

\section{List of URLs}

1. Finalists for a $\$ 10 \mathrm{~m}$ Star Trek 'tricorder' X Prize: http://www.bbc.com/news/technology28950201

2. A short video and article on the BBC that talks about the tele-journalism application of the BEAMING system: http://www.bbc.com/news/technology-18017745

3. An article published by Spanish newspaper La Vanguardia that used the system to conduct an interview: http://www.lavanguardia.com/vida/20120520/54296167703/teletransporte-barcelonalondres.html

4. The documentary aired by Catalan TV channel TV3 that showcases the BEAMING system in the context of Telejournalism: http://blogs.tv3.cat/quequicom.php?itemid=48881

5. An impromptu interview of journalist Nonny de la Peña conducted by BTV during a debate that she moderated while using the system, embodied as a humanoid robot: https://www .youtube.com/watch?v=FFaInCXi9Go

6. The article describing the interview using the system published in traditional news media (Latino LA), which focused solely on the substantive issue of food for the brain, rather than the system that was used: http://latinola.com/story.php?story=12654

\section{References}

[1] J.-M. Normand, B. Spanlang, F. Tecchia, M. Carrozzino, D. Swapp, and M. Slater, "Full Body Acting Rehearsal in a Networked Virtual Environment - A Case Study," PRESENCE - Teleoperators and Virtual Environments, vol. 21, pp. 229-243, 2012.

[2] W. Steptoe, J. M. Normand, O. Oyekoya, F. Pece, E. Giannopoulos, F. Tecchia, et al., "Acting in Collaborative Multimodal Mixed Reality Environments," PresenceTeleoperators and Virtual Environments, vol. 21, pp. 406-422, 2012. 
[3] D. Perez-Marcos, M. Solazzi, W. Steptoe, O. Oyekoya, A. Frisoli, T. Weyrich, et al., "A fully immersive set-up for remote interaction and neurorehabilitation based on virtual body ownership," Front. Neur., vol. 3:110. doi, 2012.

[4] M. V. Sanchez-Vives and M. Slater, "From Presence to Consciousness Through Virtual Reality," Nature Reviews Neuroscience, vol. 6, pp. 332-339, 2005.

[5] J. M. Normand, M. V. Sanchez-Vives, C. Waechter, E. Giannopoulos, B. Grosswindhager, B. Spanlang, et al., "Beaming into the Rat World: Enabling Real-Time Interaction between Rat and Human Each at Their Own Scale," PLoS ONE, vol. 7, p. e48331, 2012.

[6] A. Steed, W. Steptoe, W. Oyekoya, F. Pece, T. Weyrich, J. Kautz, et al., "Beaming: An Asymmetric Telepresence System," IEEE Computer Graphics and Applications, vol. 32, pp. 10-17, 2012.

[7] M. Slater, B. Spanlang, M. V. Sanchez-Vives, and O. Blanke, "First person experience of body transfer in virtual reality," PLOS ONE, vol. 5, pp. e10564-e10564, 2010.

[8] M. Minsky, "Telepresence," Omni, pp. 45-52, 1980.

[9] E. Guizzo, "When my avatar went to work," Spectrum, IEEE, 2010.

[10] K. M. Tsui and M. Desai, "Exploring use cases for telepresence robots," in 6th ACM/IEEE International Conference on Human-Robot Interaction (HRI), Lausanne, Switzerland, 2011, pp. 11-18.

[11] B. Spanlang, X. Navarro, J.-M. Normand, S. Kishore, R. Pizarro, and M. Slater, "Real time whole body motion mapping for avatars and robots," Proceedings of the 19th ACM Symposium on Virtual Reality Software and Technology - VRST '13, pp. 175-178, 2013.

[12] M. Gellhorn, The view from the ground: Atlantic Monthly Press, 1988.

[13] K. L. Nowak and C. Rauh, "Choose your "buddy icon" carefully: The influence of avatar androgyny, anthropomorphism and credibility in online interactions," Computers in Human Behavior, vol. 24, pp. 1473-1493, 2008.

[14] E. Paulos and J. Canny, "Social tele-embodiment: Understanding presence," Autonomous Robots, pp. 87-95, 2001.

[15] S. O. Adalgeirsson and C. Breazeal, "MeBot: A robotic platform for socially embodied telepresence," 2010 5th ACM/IEEE International Conference on Human-Robot Interaction (HRI), pp. 15-22, 2010.

[16] T.-C. Tsai, Y.-L. Hsu, A.-I. Ma, T. King, and C.-H. Wu, "Developing a telepresence robot for interpersonal communication with the elderly in a home environment," Telemedicine journal and e-health : the official journal of the American Telemedicine Association, vol. 13, pp. 407-24, 2007.

[17] V. I. Petkova and H. H. Ehrsson, "If I Were You : Perceptual Illusion of Body Swapping," PLoS ONE, vol. 3, p. e3832, 2008.

[18] A. Maselli and M. Slater, "The building blocks of the full body ownership illusion," Frontiers in Human Neuroscience, vol. 7, 2013. 
[19] J. M. Normand, E. Giannopoulos, B. Spanlang, and M. Slater, "Multisensory Stimulation Can Induce an Illusion of Larger Belly Size in Immersive Virtual Reality," PLoS ONE, vol. 6, p. e16128. doi:10.1371/journal.pone.0016128, 2011.

[20] D. Banakou, R. Groten, and M. Slater, "Illusory ownership of a virtual child body causes overestimation of object sizes and implicit attitude changes," PNAS, vol. 110, pp. 12846$12851,2013$.

[21] T. C. Peck, S. Seinfeld, S. M. Aglioti, and M. Slater, "Putting yourself in the skin of a black avatar reduces implicit racial bias.," Consciousness and cognition, vol. 22, pp. 779$787,2013$.

[22] K. Kilteni, I. Bergstrom, and M. Slater, "Drumming in immersive virtual reality: the body shapes the way we play.," IEEE transactions on visualization and computer graphics, vol. 19, pp. 597-605, 2013.

[23] L. Maister, N. Sebanz, G. Knoblich, and M. Tsakiris, "Experiencing ownership over a dark-skinned body reduces implicit racial bias," Cognition, vol. 128, pp. 170-178, 2013.

[24] K. Kilteni, J.-M. Normand, M. V. Sanchez Vives, and M. Slater, "Extending Body Space in Immersive Virtual Reality: A Very Long Arm Illusion," PLoS ONE, vol. 7, p. e40867, 2012.

[25] M. Tsakiris, L. Carpenter, D. James, and A. Fotopoulou, "Hands only illusion: multisensory integration elicits sense of ownership for body parts but not for noncorporeal objects," Experimental Brain Research, pp. 1-10, 2009.

[26] S. Kishore, M. González-Franco, C. Hintemüller, C. Kapeller, C. Guger, M. Slater, et al., "Comparison of SSVEP BCI and Eye Tracking for Controlling a Humanoid Robot in a Social Environment," PRESENCE: Teleoperators and Virtual Environments, vol. 23, pp. 242-252, 2014.

[27] O. Blanke and T. Metzinger, "Full-body illusions and minimal phenomenal selfhood," Trends in Cognitive Sciences, vol. 13, pp. 7-13, 2009.

[28] M. Tsakiris, G. Prabhu, and P. Haggard, "Having a body versus moving your body : How agency structures body-ownership," Consciousness and Cognition, vol. 15, pp. 423-432, 2006.

[29] M. V. Sanchez-Vives, B. Spanlang, A. Frisoli, M. Bergamasco, and M. Slater, "Virtual hand illusion induced by visuomotor correlations," PLOS ONE, vol. 5, p. e10381, 2010.

[30] E. Kokkinara and M. Slater, "Measuring the effects through time of the influence of visuomotor and visuotactile synchronous stimulation on a virtual body ownership illusion," Perception, vol. 43, pp. 43 - 58, 2014.

[31] D. Banakou and M. Slater, "Body Ownership Causes Illusory Self-Attribution of Speaking and Influences Subsequent Real Speaking," PNAS, vol. 111, pp. 17678-17683, 2014.

[32] S. Nishio, K. Taura, H. Sumioka, and H. Ishiguro, "Effect of Social Interaction on Body Ownership Transfer to Teleoperated Android," ROMAN 2013 - The 23rd IEEE International Symposium on Robot and Human Interactive Communication, pp. 565-570, 2013. 
[33] K. Ogawa, K. Taura, S. Nishio, and H. Ishiguro, "Effect of perspective change in body ownership transfer to teleoperated android robot," 2012 IEEE RO-MAN: The 21st IEEE International Symposium on Robot and Human Interactive Communication, pp. 10721077, 2012.

[34] F. Tecchia, M. Carrozzino, S. Bacinelli, F. Rossi, D. Vercelli, G. Marino, et al., "A Flexible Framework for Wide-Spectrum VR Development," Presence - Teleoperators and virtual Environments, vol. 19, pp. 302-312, 2010.

[35] M. Slater, "Place Illusion and Plausibility can lead to realistic behaviour in immersive virtual environments," Philos Trans R Soc Lond, vol. 364, pp. 3549-3557, 2009.

[36] P. Kaliman and M. Aguilar, Cocina para tu mente: Blume, 2014. 
Transporter
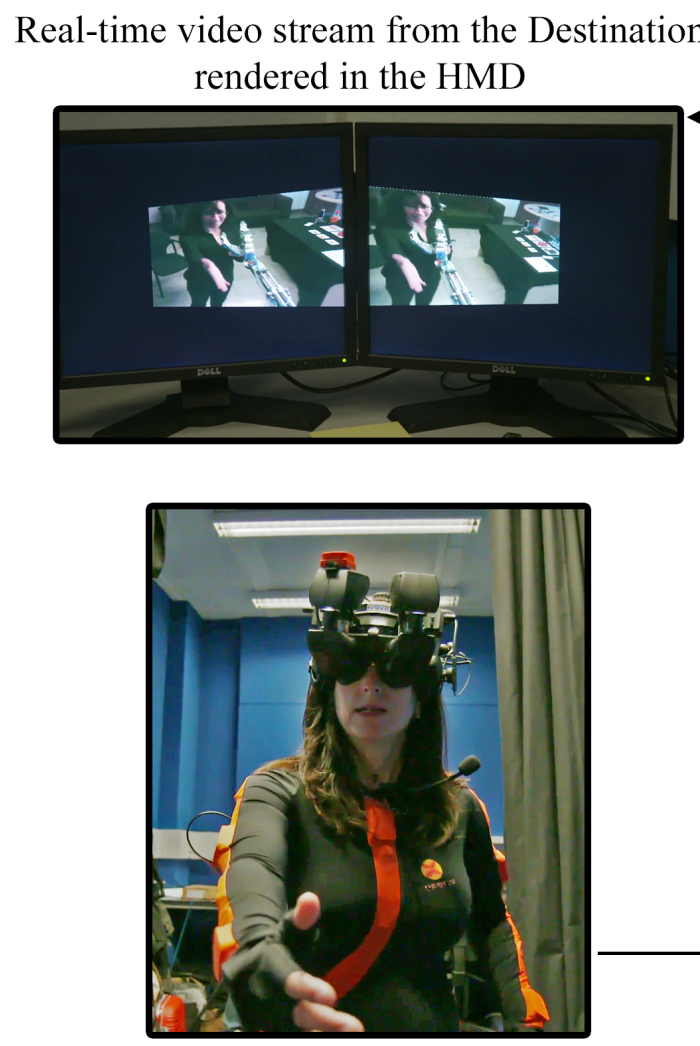

Visitor

\section{Destination}

Capture and stream

Destination information

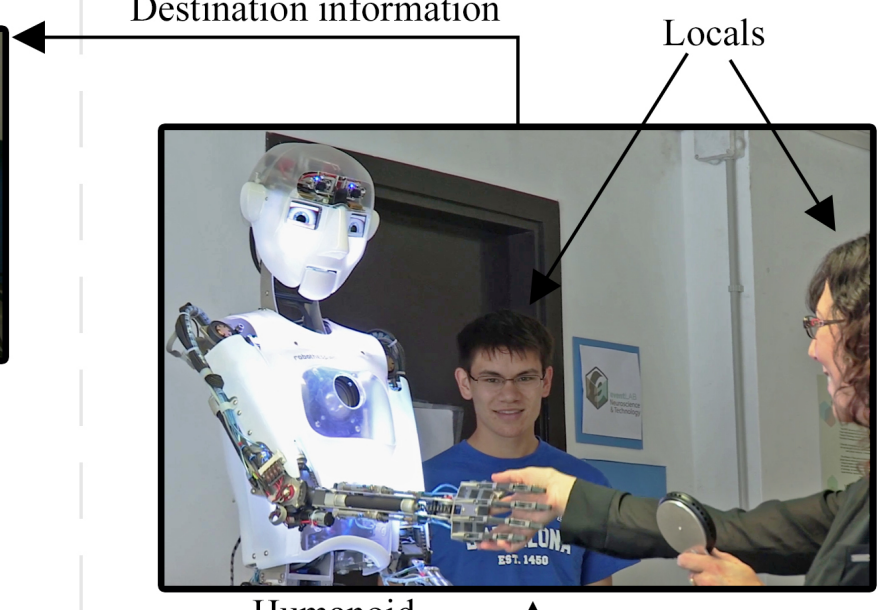

Humanoid

Robot

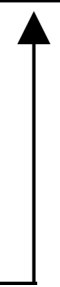

Capture and stream

Visitor information

Figure 1: Flow and exchange of information between the transporter and destination. The visitor wears a body-tracking suit through which the robot is controlled, while the HMD displays the stereo camera feed from the destination. 


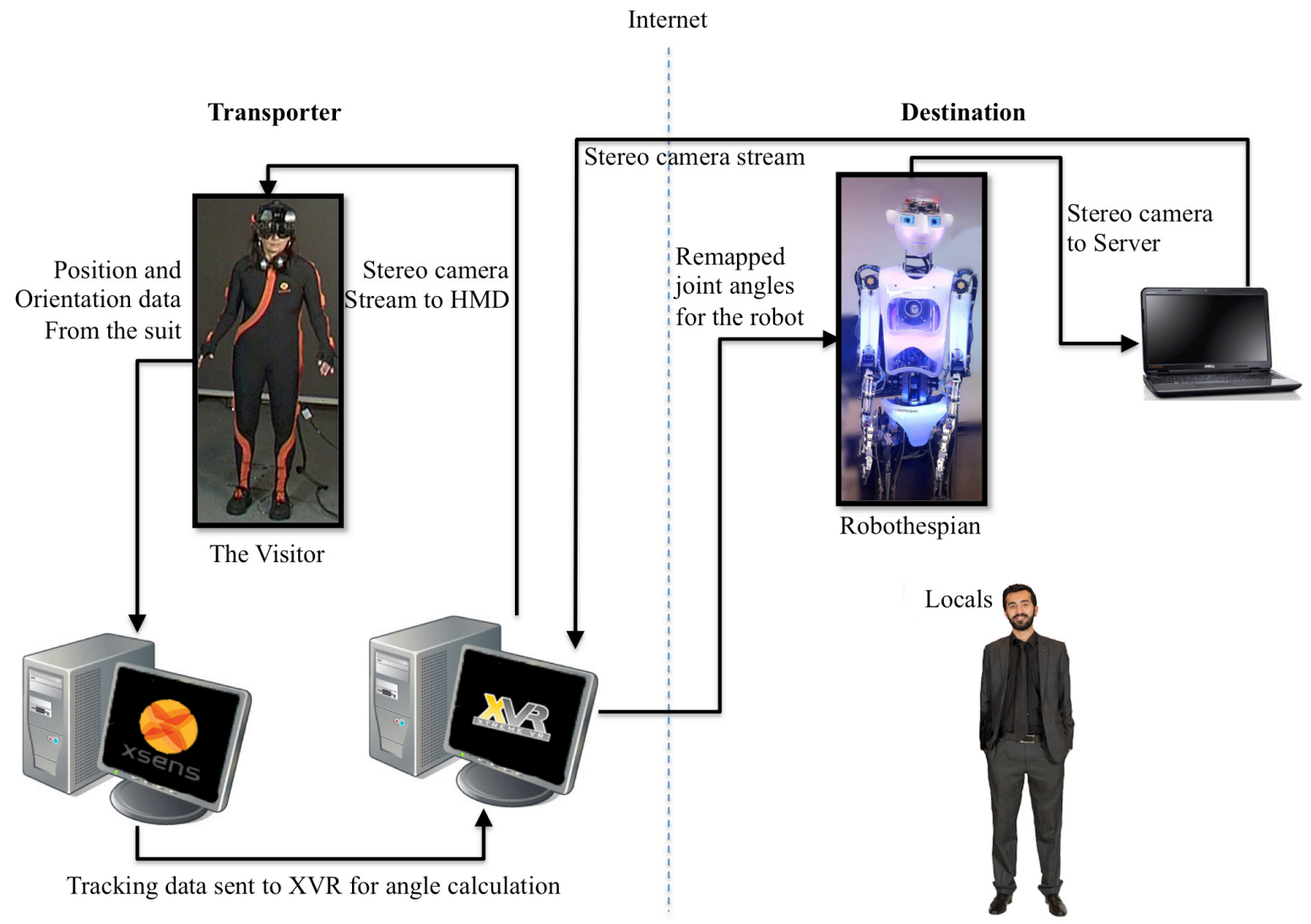

Figure 2: The specification of the setup used for the immersive journalism interview. Journalist Nonny de la Peña wore an Xsens body tracking suit and an NVIS nVisor SX111 HMD. At the destination she was represented by a custom-built Robothespian with two Microsoft HD-3000 webcams separated at a standard interocular distance, manufactured by Engineered Arts. The entire communication and exchange of data took place through a regular Internet connection. 